\title{
ON THE LOSS OF ANGULAR MOMENTUM FROM STARS IN THE PRE-MAIN SEQUENCE PHASE
}

\author{
ISAO OKAMOTO \\ The International Latitude Observatory of Mizusawa, Iwate, Japan
}

(Paper read by $K$. Nariai)

\begin{abstract}
The braking of stellar rotation in the wholly convective phase in the pre-main sequence is numerically discussed. The structure of stars in that phase is expressed by a rotating polytrope with an index of 1.5 and the Schatzman-type mechanism is used as the means of loss of angular momentum. The magnetic energy is assumed to change with evolution as $H_{0}{ }^{2} / 8 \pi\left(R / R_{0}\right)^{8}$, where $H_{0}$ and $R_{0}$ are initial magnetic field and radius, and $s$ is a free parameter. The changes of angular momentum, rotational velocity, etc. with contraction are calculated from the initial state, which is taken to be the state when the stars flared up to the Helmholtz-Kelvin contraction. It is shown that the exponent $s$ must be in the range from -1 to -3 so that the stars with adequate strength of the initial magnetic field may lose almost all of their angular momenta in a suitable rate if they are initially in the state of rotational instability.

Stellar rotation from the time of star formation to the main sequence stage is discussed. Also, the formation of the solar system and other planetary systems is discussed, with respect to the braking.
\end{abstract}

\section{Introduction}

It is well known that the axial rotation of the main sequence stars stops quite abruptly at about F5. Schatzman (1962) suggested an efficient mechanism due to mass loss through stellar magnetic activity related to the surface convection zone. Mestel (1968a, b) constructed an elaborate theory about magnetic braking by a stellar wind. Nariai (1968) discussed braking of stellar rotation in terms of acoustic energy supplied to coronae. Huang $(1965 \mathrm{~b}, 1967)$ investigated rotational behavior of the main sequence stars and its plausible consequences concerning formation of planetary systems.

It was shown by Hayashi (1961) that the stars in the pre-main sequence contraction stage are wholly-convective and contract downward almost vertically on the H-R diagram until the radiative core develops appreciably. Poveda (1964) presented a theory of flare stars on the basis of Schatzman's theory and calculations of stellar evolution by Hayashi and his collaborators (1962, 1963). Huang (1965a) considered the sequence of events in the early phase of the solar system and suggested that Schatzman's mechanism operated in the wholly convective phase and the sun thereby lost its angular momentum.

We connect Schatzman's loss mechanism to the theory of evolution of Hayashi et al. and calculate numerically the variation of angular momentum of the sun and stars with their evolution.

\section{The Rotation of Stars in the Wholly Convective Phase}

The angular momentum and equatorial velocity of a wholly convective star with the 
polytropic index of 1.5 are expressed by the following relations;

and

$$
\omega C=(\omega C)_{A} \sqrt{G M^{3} R}
$$

$$
v_{\mathrm{eq}}=\omega(A) \sqrt{\frac{G M}{R},}
$$

where $A=\omega^{2} / 8 \pi G \underline{\varrho}_{c}$ (see Okamoto (1969) (Paper I) for the derivation of Equations (1) and (2)). The dimensionless quantities $\omega(A)$ and $(\omega C)_{A}$ are functions of $A$ only and shown in Figure 1. The rotation equation which contains both terms of gravitational

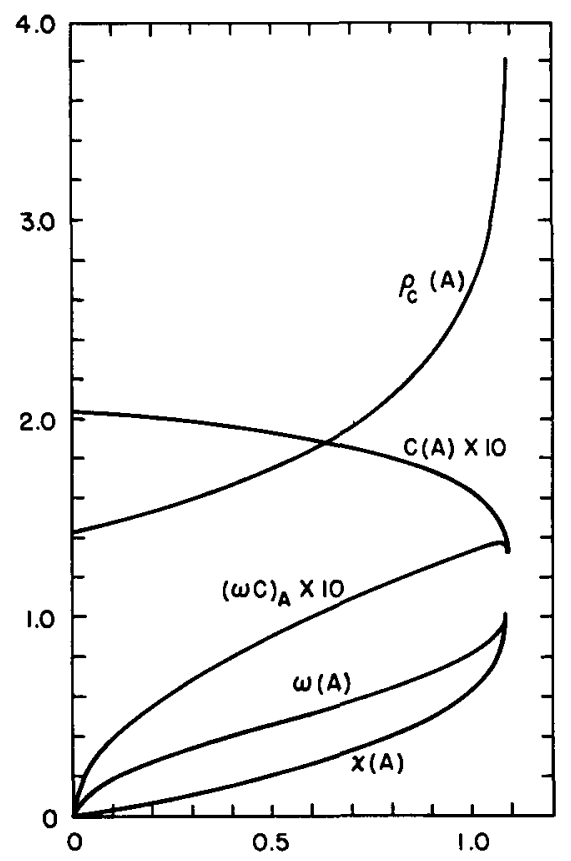

Fig. 1. $C(A), \rho_{\mathrm{c}}(A), \omega(A),(\omega C)_{A}$ and $\chi(A)$ are plotted as functions of $A$ for the polytrope with $n=1.5$. The values multiplied by a factor 10 are plotted for $C(A)$ and $(\omega C)_{A}$.

contraction and magnetic braking is obtained, by equating the time derivative of $\omega C$ to the magnetic torque, as follows;

$$
\frac{\mathrm{d}}{\mathrm{d} A}(\omega C)_{A} \cdot \frac{\mathrm{d} A}{\mathrm{~d} t}=(\omega C)_{A} \cdot \frac{1}{2}\left(-\frac{1}{R} \frac{\mathrm{d} R}{\mathrm{~d} t}\right)+\frac{1}{\sqrt{G M^{3} R}} \frac{1}{4 \pi} \int H_{\phi} r \sin \theta(\mathbf{H} \mathrm{d} \mathbf{S}) .
$$

The Helmholtz-Kelvin contraction time is expressed in terms of the stellar luminosity;

$$
\left(-\frac{1}{R} \frac{\mathrm{d} R}{\mathrm{~d} t}\right)^{-1}=\frac{3 \gamma-4}{3(\gamma-1)} \cdot \frac{3}{5-n} \cdot \frac{G M^{2}}{R L}=\frac{3}{7} \frac{G M^{2}}{R L},
$$

where the values $n=1.5$ and $\gamma=\frac{5}{3}$ are inserted. For the mechanism of magnetic braking we use a modified form of the Schatzman-type. Then, we can equate approximately 
the second term of Equation (3) in the following way;

$$
\begin{gathered}
1 \\
4 \pi
\end{gathered} \int H_{\phi} r \sin \theta(\mathbf{H} \mathrm{d} \mathbf{S})=\frac{\mathrm{d} M}{\mathrm{~d} t} \omega r_{\mathrm{c}}^{2} \quad\left(\mathrm{~g} \cdot \mathrm{cm}^{2} \cdot \mathrm{sec}^{-1} / \text { years }\right),
$$

where mass loss $\mathrm{d} M / \mathrm{d} t$ and critical distance $r_{\mathrm{c}}$ are given by the following Equations (6) and (7) respectively.

$$
\begin{array}{rl}
\frac{\mathrm{d} M}{\mathrm{~d} t}=-4 & 93 \\
\times 10^{32} \times \frac{H_{*}^{2}}{4 \pi V^{2}}\left(\frac{L}{L_{0}}\right)^{1 / 2}\left(\begin{array}{c}
M_{0} \\
M_{\odot}
\end{array}\right)^{19 / 24} \\
\times\left(\frac{R_{0}}{R_{\odot}}\right)^{27 / 24}\left(\frac{M}{M_{0}}\right)^{-5 / 24}\left(\frac{R}{R_{0}}\right)^{53 / 24} \omega(A)^{3 / 4} \quad \text { (g/years) }
\end{array}
$$

and

$$
r_{\mathrm{c}}=R\left[\begin{array}{cc}
1 & 1 \\
\sqrt{2} & \omega(A)
\end{array}\left(\frac{V_{A}}{V}\right)^{2}\right]^{1 / 3}
$$

The velocity of ejected matter and its change along the evolutionary path cannot be definitely determined from observations and theory. Therefore, for simplicity, we take the escape velocity of the star for the ejection velocity of matter;

$$
V=V_{\mathrm{esc}}=\left(\begin{array}{c}
2 G M \\
R
\end{array}\right)^{1 / 2}=617.7\left(\begin{array}{c}
M \\
M_{\odot}
\end{array}\right)^{1 / 2}\left(\frac{R}{R_{\odot}}\right)^{-1 / 2} \mathrm{~km} / \mathrm{sec}
$$

The initial radii of stars when they flare up from the pre-opaque dynamical contraction and reach the state of quasi-static contraction are given by the relation

$$
\frac{13}{25-n}-\frac{G M^{2}}{R}=\chi_{m_{\mathrm{H}}}^{M}
$$

where $m_{H}$ is the mass of the hydrogen atom and $\chi=15.84 X+19.75 Y, X$ and $Y$ being the concentrations by mass of hydrogen and helium. If we take the stars of Population I, putting $X=0.61$ and $Y=0.37$, we obtain the initial radii in terms of stellar mass as follows;

$$
R / R_{\odot}=49.88\left(M / M_{\odot}\right)
$$

Then, from Equation (2) the rotational velocity at the initial radius (9) depends only upon $A$ and not upon the stellar mass. The radius given by Equation (9), however, corresponds to the non-rotating state. In order to carry out the integration of Equations (3) and (4), we must know the initial value of $A$ also. But we are not able to determine both $R_{0}$ and $A_{0}$ at the same time. Thus, we assume the value of $1.069 \times 10^{-2}$ for $A_{0}$, at which stars are near to the limit of rotational instability.

The path of the evolution from the initial state (9) is approximated by the following relation

$$
L / L_{\odot}=Q\left(R / R_{\odot}\right)^{1.5}
$$

where $Q=0.154,0.269,0.624$ and 1.161 for stars of $0.4,0.6,1.0$ and $2.0 M_{\odot}$, respectively. The radii where radiative cores just begin to grow at the centers are $0.776,1.318$, 
2.570 and 11.75 in units of the solar radius for stars of these masses (see Table I in Okamoto (1967)). Though Iben (1965) has calculated the evolutionary paths of the pre-main sequence stars in detail, the paths themselves do not have an important effect upon the present calculations. Also, the effects of rotation and the magnetic field are not taken into account in his calculations. Thus, the approximate relation (10) is sufficient for our purpose.

Since the magnetic energy appears in the equations for $\mathrm{d} M / \mathrm{d} t$ and $r_{\mathrm{c}}$, we need knowledge of the change of stellar magnetic field with evolution. But we do not know accurately the origin of the stellar magnetic field and its change with evolution at present. Here we consider the dynamo mechanism in the stellar convection zone (Parker, 1955) and assume for the change with evolution the following:

$$
\frac{H_{*}^{2}}{8 \pi}=\frac{H_{0}^{2}}{8 \pi}\left(\frac{R}{R_{0}}\right)^{s},
$$

where $H_{0}$ is the initial field at $R=R_{0}$. If the magnetic field is frozen in the stellar material during the contraction, we should take $s=-4$. Provided that the primordial

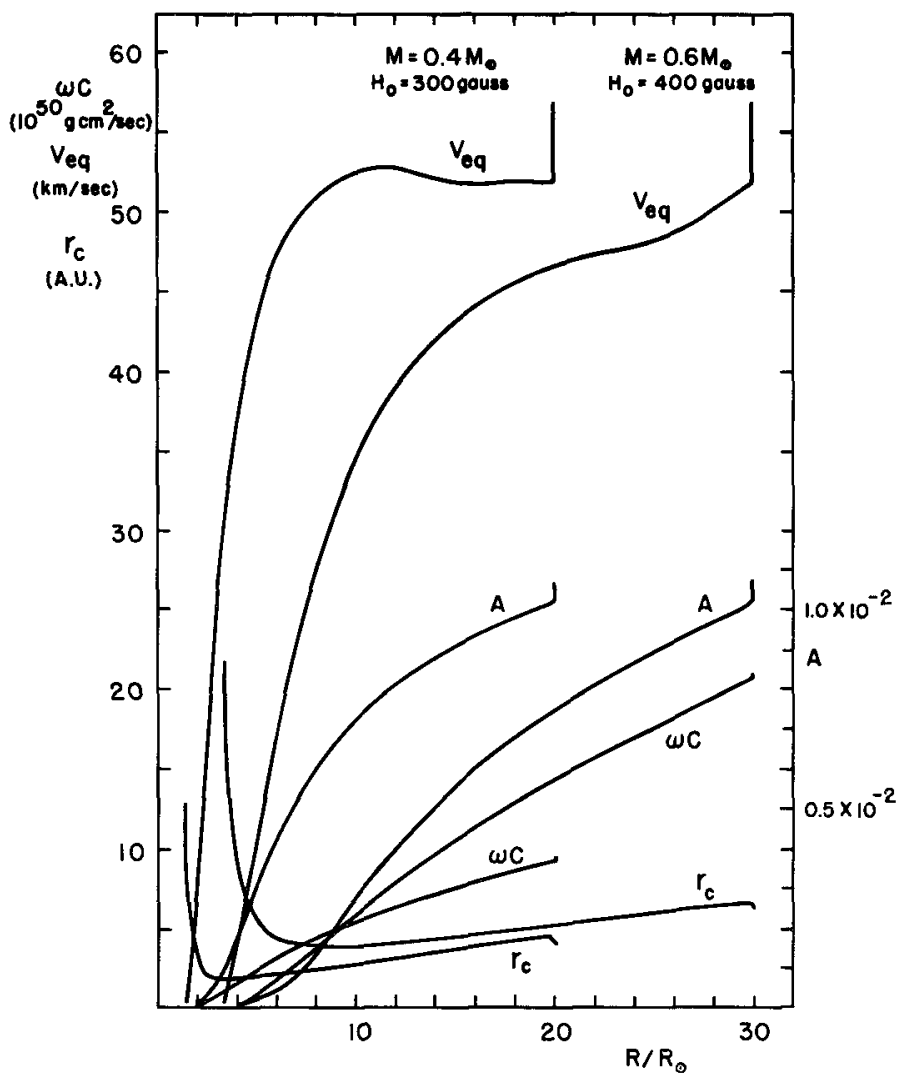

Fig. 2. The change of angular momentum $\omega C$, equatorial velocity $V_{\text {eq }}$, critical distance $r_{\mathrm{c}}$ and the parameter $A$ with the contraction of the stars of 0.4 and $0.6 M_{\odot}$ for $H_{0}=300$ and $400 \mathrm{G}$, respectively, when $s=-1$. 
field decays with contraction, $s$ is positive. We take $-4 \leqslant s \leqslant 1$ in the following numerical calculations.

Numerical integrations of simultaneous differential Equations (3) and (4) are carried out, starting from the initial radius (9) through the evolutionary path (10), where relations (5), (6), (7) and (8) are used. If we know $R$ and $A$ through integrations, we can know the angular momentum $\omega C$ and the equatorial velocity $V_{\text {eq }}$ of the star from Equations (1) and (2). The quantities $A, \omega C, V_{\text {eq }}$ and $r_{\mathrm{c}}$ are plotted in Figures 2 and 3 as functions of the radius in terms of the solar radius. For $s=-1$ we take $H_{0}=300 \mathrm{G}$ for $0.4 M_{\odot}, H_{0}=400 \mathrm{G}$ for $0.6 M_{\odot}, H_{0}=400 \mathrm{G}$ for $1.0 M_{\odot}$ and $H_{0}=600 \mathrm{G}$ for $2.0 M_{\odot}$ (see Okamoto (1969) for results for other values of $s$ and $H_{0}$ ). Figures 2 and 3 show the sharp decreases of $A, \omega C, V_{\text {eq }}$ at the initial state. It is because $(\omega C)_{A}$ has the maximum value at the point $A_{\max }=1.08 \times 10^{-2}$ slightly smaller than $A_{c r}=1.0906$, where the rotational instability sets in according to James (1964), and $\mathrm{d}(\omega C)_{A} / \mathrm{d} A=0$ at $A=A_{\max }$. If the right-hand side of Equation (3) is non-zero, we have $\mathrm{d} A / \mathrm{d} t= \pm \infty$. We have avoided the maximum of $(\omega C)_{A}$ in the numerical calculation and selected

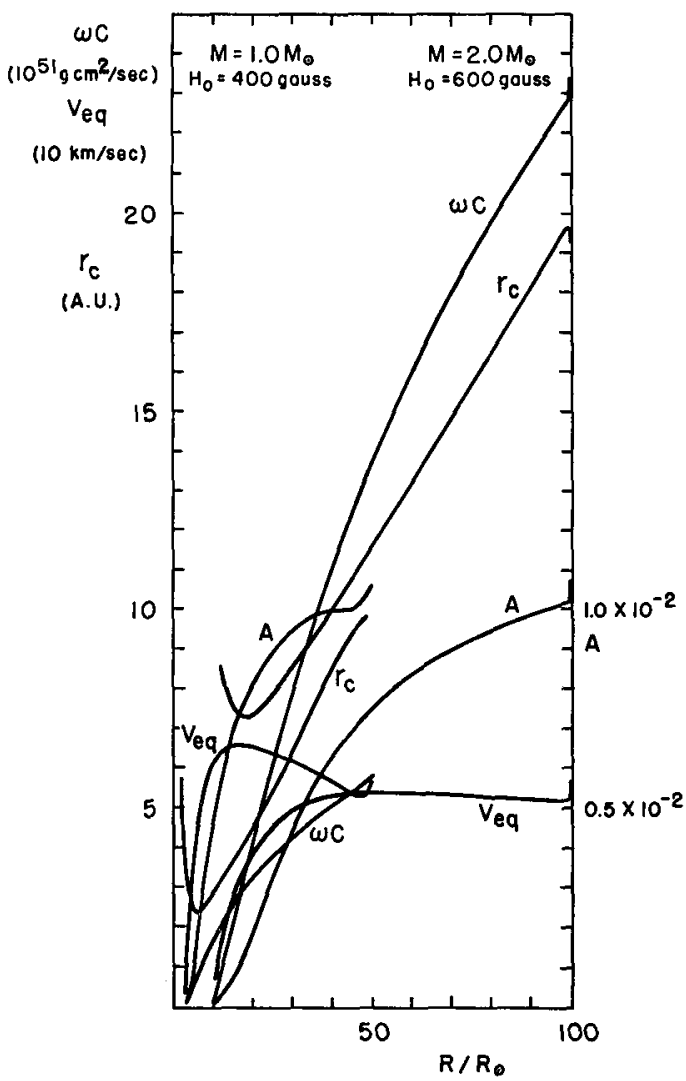

Fig. 3. The change of angular momentum $\omega C$, equatorial velocity $V_{\text {eq }}$, critical distance $r_{\mathrm{c}}$ and the parameter $A$ with the contraction of the stars of 1.0 and $2.0 M_{\odot}$ for $H_{0}=400$ and $600 \mathrm{G}$, respectively, when $s=-1$. 
$1.069 \times 10^{-2}$ instead of $A_{c r}$ for the initial value of $A$. However, $\mathrm{d}(\omega C)_{A} / \mathrm{d} A$ is still so small that $A, \omega C$ and $V_{\text {eq }}$ show a rapid decrease.

In the above calculations, we have taken the escape velocity of a non-rotating star for the ejection velocity of matter due to flare activity. The escape velocity of a rapidly rotating star is considerably smaller than given by Equation (8), owing to the centrifugal force. Then, as we see from Equations (6) and (7), this mechanism for throwing out stellar angular momentum would be much more effective.

The ratios of the total mass loss necessary to throw away almost all the stellar angular momenta to the stellar mass are smaller than, or at most equal to $2 \times 10^{-4}$. This degree of mass loss lies well within the range deduced from observations of $\mathrm{T}$ Tauri stars (Kuhi, 1964, 1966).

We can also guess the change of magnetic energy of stars in the wholly convective phase from the braking of stellar rotation. The protostars will have a reasonable strength of the initial field $H_{0}$ resulting from the interstellar magnetic field. Then, from the calculations we know that the exponent $s$ must be negative for protostars to be braked during the wholly convective phase. We suggest a value from -1 to -3 for $s$ so that the protostars may be braked with a suitable speed.

\section{Discussion}

We now conjecture about the rotations of stars from the time of star formation to the main sequence stage. According to recent theories of star formation (Mestel, 1965), an interstellar cloud starts contracting owing to gravitational instability and protostars are formed by fragmentation of a collapsing cloud. The subsequent evolution is divided into two main phases, one is the phase of dynamical contraction which proceeds more or less rapidly after star formation, and the other is the following phase of slow contraction (Helmholtz-Kelvin contraction) toward the main sequence (Hayashi, 1966).

In the previous section we assumed that stars are near the limit of rotational instability when they flare up from the state of dynamical contraction to the Hayashi phase and we adopted $1.069 \times 10^{-2}$ for $A_{0}$. At present we have little knowledge about the rotational state of protostars. Struve (1945) and Huang and Struve (1954), however, showed from a statistical study that rotating stars have not acquired their angular momentum from the galactic rotation of the prestellar gaseous medium and their angular momenta must have been derived from a random process, such as a random spin in fragmentation of a collapsing cloud. The angular momentum and the magnetic field may be formidable obstacles for condensation of new stars. Here we assume that protostars may succeed in evolving into the pre-opaque dynamical contraction stage. The magnetic energy of a gravitationally-bound condensation can never exceed the gravitational energy. Thus, the interstellar magnetic field will not be able to become strong enough to transfer all of the stellar angular momentum. As the contraction proceeds, the rotational instability may take place in this stage and break up equatorially to shed the stellar matter. Therefore, when the protosun and protostars flare up 
to the stage of quasihydrostatic equilibrium, they will be at least nearly rotationally unstable.

The angular momentum of the solar system $\left(3.156 \times 10^{50} \mathrm{~g} \cdot \mathrm{cm}^{2} / \mathrm{sec}\right)$ is possessed almost entirely by the orbital motion of the giant planets, with the sun having only $0.5 \%$ of the whole as its spin angular momentum. If the angular momentum of the present solar system had concentrated in the sun when it flared up, we obtain $A_{0}=$ $0.008 \times 10^{-2}$ and the ratio of the centrifugal force to gravity at the equator $\chi\left(A_{0}\right)=$ 0.003 from Figure 1. If these values for the protosun were true, the protosun would have had to suffer a great degree of braking during the dynamical contraction stage. As discussed above, however, the magnetic field could not effectively brake the protosun to the state of such a slow rotation during this stage. Thus, from Equations (1) and (9) and using the value of $1.069 \times 10^{-2}$ for $A_{0}$, the protosun had the values of $58 \times 10^{50} \mathrm{~g} \cdot \mathrm{cm}^{2} / \mathrm{sec}$ as the angular momentum at the moment of the flare-up, which is about twenty times larger than the present value of the solar system. The chemical composition of the protoplanetary cloud would be the same as that of the sun, and the lower limit on the initial mass of the cloud is estimated by diluting planetary material with volatile substances until the solar composition is reached. The extra mass over the present total mass of the planets would be lost out of the solar system because of the increasing centrifugal force that resulted as the protosun began to transfer its angular momentum.

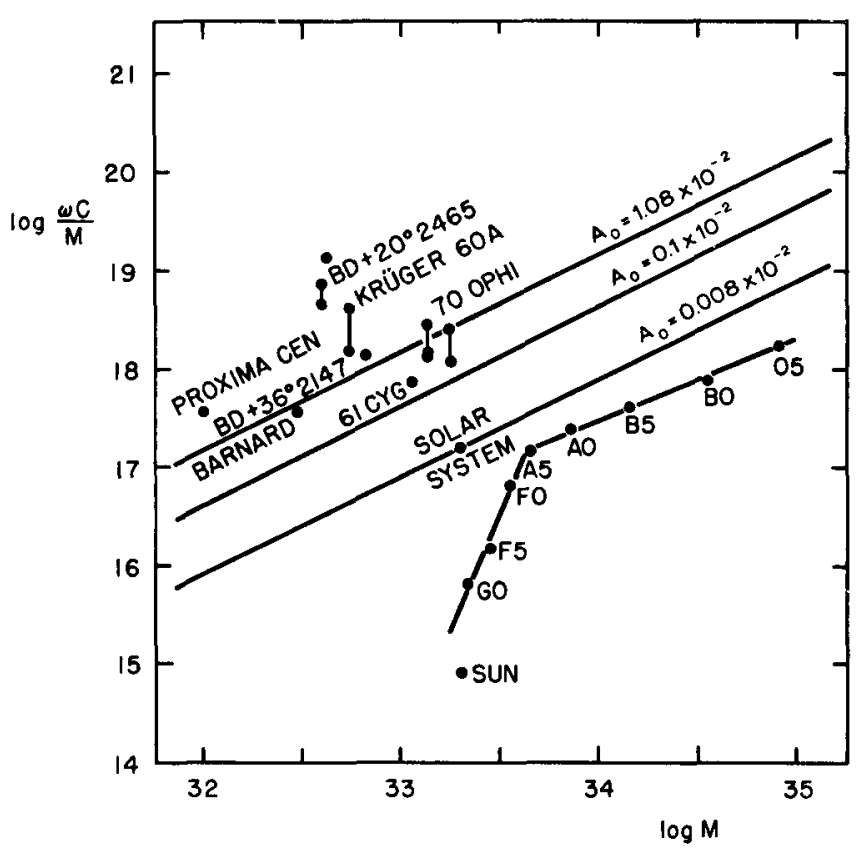

Fig. 4. The logarithm of angular momentum per unit mass versus the logarithm of stellar mass. The values for the main sequence stars are taken form McNally (1965) and the values for the "planetary systems' from van den Heuvel (1966). 
We plot in Figure 4 the logarithm of the stellar angular momentum per unit mass versus the logarithm of the stellar mass. The straight line $A_{0}=1.08 \times 10^{-2}$ is drawn from Equations (1) and (9) and shows the angular momentum per unit mass at the limit of the rotational instability. The line $A_{0}=0.008 \times 10^{-2}$ corresponds to the case where the present total angular momentum of the solar system is concentrated in the protosun at the flare-up. We also plot the angular momentum per unit mass for the main sequence stars from the calculation of McNally (1965). The line for $A_{0}=1.08 \times$ $10^{-2}$ and the curve for the main sequence stars of early type are nearly parallel. The difference between both lines shows that even early type stars suffered a considerable degree of braking. Actually, as Huang (1965b) suggested, the braking is not limited only to stars of spectral type later than F5, but extends to O, B, A and early F stars as well. These early-type stars are considered to have suffered braking during the Hayashi phase and no braking afterwards because of the retirement of the surface convection zone in the succeeding post-Hayashi phase. Further contraction caused them to rotate rapidly because of conservation of angular momentum. The slow rotation of late-lype stars, including the sun, may be regarded as a result of their having continued to receive braking from the wholly convective phase to the main sequence.

Finally we discuss the possible occurrence of planetary systems around stars. Huang $(1965,1967)$ suggested that the formation of planetary systems is closely related to the braking of stellar rotation, and defined the following three characteristics for any planetary system; (1) a small mass ratio of the total mass of the entire planetary system to the mass of the central star; (2) a large angular momentum; (3) the majority of its members moving in nearly coplanar and circular orbits. In Figure 4 we plot the angular momentum per unit mass for 'planetary systems' from van den Heuvel's paper (1966). While more than half of them lie beyond the line for $A_{0}=1.08 \times 10^{-2}$, the remainder are near to that line. These facts imply that contrary to the case for the solar system these 'planetary systems' have a nearly equal or larger amount of angular momentum than the angular momentum of the central stars at their flare-up. They seem to have angular momentum too large for planetary systems. This is because the mass ratio of 'planets' to the central stars in Table I of van den Heuvel's paper are more than an order of magnitude larger than the mass ratio of the solar system. Also, Kumar (1964) and Huang (1967) emphasized that the unseen companion of Barnard's star inferred by van de Kamp (1963) from the proper motion should not be regarded as forming a planetary system, because the eccentricity is equal to 0.6 , a fairly large value. Consequently van den Heuvel's 'planetary systems' are different from the definition of Huang for planetary systems. These may be regarded as intermediate between binary systems and planetary systems. Nevertheless we expect that planetary systems must be common in space. We may not as yet have discovered them with today's instruments.

\section{Acknowledgements}

The author wishes to thank Dr. K. Nariai for reading this manuscript at the Colloquium on Stellar Rotation. His thanks are also due to Dr. T. Osaki for suggesting to him 
that the paper be sent to the Colloquium and to Professor Slettebak for various arrangements.

\section{References}

Hayashi, C.: 1961, Publ. Astron. Soc. Japan. 13, 450.

Hayashi, C.: 1966, Annual Rev. Astron. Astrophys. 4, 171.

Hayashi, C. and Nakano, T.: 1963, Prog. Theoret. Phys. 30, 460.

Hayashi, C., Hoshi, R., and Sugimoto, D.: 1962, Prog. Theoret. Phys. Suppl. 22, 165.

Huang, S.-S.: 1965a, Publ. Astron. Soc. Pacific. 77, 42.

Huang, S.-S.: 1965b, Astrophys. J. 141. 985.

Huang, S.-S.: 1967, Astrophys. J. 150, 229.

Huang, S.-S. and Struve, O.: 1954, Ann. Astrophys. 17, 85.

Iben, I.: 1965, Astrophys. J. 141, 993.

James, R. A.: 1964, Astrophys. J. 140, 552.

Kuhi, L. V.: 1964, Astrophys. J. 140,1409.

Kuhi, L. V.: 1966, Astrophys. J. 143, 991.

Kumar, S. S.: 1964, Z. Astrophys. 58, 248.

McNally, D.: 1965, Observatory 85, 166.

Mestel, L.: 1965, Quart. J. Roy. Astron. Soc. 6, 161, 265.

Mestel, L.: 1968a, Monthly Notices Roy. Astron. Soc. 138, 359.

Mestel, L.: 1968b, Monthly Notices Roy. Astron. Soc. 140, 177.

Nariai, K.: 1968, Astrophys. Space Sci. 3, 150.

Okamoto, I.: 1967, Publ. Astron. Soc. Japan 19, 384.

Okamoto, I.: 1968, Publ. Astron. Soc. Japan 20, 25.

Okamoto, I.: 1969, Publ. Astron. Soc. Japan 21, 350.

Parker, E. N.: 1955, Astrophys. J. 122, 293.

Poveda, A.: 1964, Nature 202, 1319.

Schatzman, E.: 1962, Ann. Astrophys. 25, 18.

Struve, O.: 1945, Pop. Astr. 53, 201, 259.

Van den Heuvel, E. P. J.: 1966, Observatory 86, 113.

Van de Kamp, P.: 1963, Astron. J. 68, 515. 